\title{
Generation of analytic semigroups and domain characterization for degenerate elliptic opera- tors with unbounded coefficients arising in fi- nancial mathematics. Part II
}

\author{
Massimiliano Giuli, Fausto Gozzi, Roberto Monte and Vincenzo \\ Vespri
}

\begin{abstract}
This paper is devoted to study the generation of analytic semigroup for a family of degenerate elliptic operators (with unbounded coefficients) which includes well known operators arising in mathematical finance. The generation property is proved by assuming some compensation conditions among the coefficients and applying a suitable modification of the techniques developed in [16]. Using the results proved in [11] concerning the generation in the space $L^{2}\left(\mathbb{R}^{d}\right)$, we prove the generation results in $L^{p}\left(\mathbb{R}^{d}\right)$ for $p \in[1,+\infty]$. These results have several consequences in connection with the financial applications [3, 11].
\end{abstract}

Mathematics Subject Classification (2000). 47D06, 35J70, 35K65, 47N10.

Keywords. generation of analytic semigroup, second order degenerate partial differential equations of elliptic and parabolic type, localization method.

\section{Introduction}

In this paper we study the generation of analytic semigroups in $L^{p}\left(\mathbb{R}^{d}\right)$, with $p$ in $[1,+\infty]$ for a family of degenerate elliptic operators with unbounded coefficients. These results can be employed to obtain existence, uniqueness and regularity estimates for the solutions of the associated (linear or semilinear) parabolic problems, through the well known theory of analytic semigroups (e.g. [12]). This has been done in [3] for the so-called "no-arbitrage" operators arising in pricing contingent 
claims. We consider the following differential operator in $\mathbb{R}^{d}$

$$
\mathcal{A}(x, D)=\sum_{i, j=1}^{d} \psi_{i}(x) \psi_{j}(x) a_{i, j}(x) D_{i, j}+\sum_{i=1}^{d} b_{i}(x) D_{i}-\gamma^{2}(x)
$$

(denoted simply by $\mathcal{A}$ in the following), where the weights $\psi_{i}: \mathbb{R}^{d} \mapsto \mathbb{R}, i=$ $1, \ldots, d$, are differentiable sublinear functions vanishing in not more than a negligible set $Z$, the matrix $\left\{a_{i, j}\right\}_{i, j=1, \ldots, d}$ is bounded and uniformly elliptic, the coefficients $b_{i}: \mathbb{R}^{d} \mapsto \mathbb{R}, i=1, \ldots, d$, are measurable functions and the function $\gamma: \mathbb{R}^{d} \mapsto \mathbb{R}$ is differentiable and locally square integrable with its first derivatives. The main difficulty to overcome here is the need of managing both the possible unboudedness of all the coefficients and the presence of zero's for those of the second order terms. In general these operators do not generate analytic nor strongly continuous semigroups (for instance the Ornstein-Uhlenbeck operator in one dimension, where $\psi=1, b(x)=x, \gamma=0)$. However, we prove that choosing suitable compensation conditions on the coefficients this become possible.

In [11] we considered the operator $\mathcal{A}$ defined in the whole space $\mathbb{R}^{d}$ and we proved the generation of analytic semigroup in the space $L^{2}\left(\mathbb{R}^{d}\right)$, by an application of Hilbert space techniques. This was possible thanks to some preliminary a priori estimates, which are established by an appropriate choice of some compensation conditions among the coefficients of the operator. Then we obtained a characterization of the domain of the operator in $L^{2}\left(\mathbb{R}^{d}\right)$ by a localization procedure which was adapted to the growth rate of the weights $\psi$ 's at infinity and close to the negligible set $Z$ of all zeros of the $\psi$ 's.

The aim of this paper is to pass from the $L^{2}\left(\mathbb{R}^{d}\right)$ case to the $L^{p}\left(\mathbb{R}^{d}\right)$ one, when $1 \leq p \leq+\infty$, by using a suitable modification of the Stewart's method $[15,16,17]$. Of course the fitting localization procedure become more complicated here, since it now depends also on the growth rate of the zero order coefficient $\gamma$.

A first result of our semigroup generation analysis is the existence of solutions of the no-arbitrage pricing problems, which is a central topic in the modern mathematical finance. However, a general existence result can be obtained via the probabilistic approach. So the main motivation to study these generation problems is based on the the question of regularity of solutions. This also in order to apply suitable numerical methods.

The paper is organized as follows. In Section 2 we introduce the notation and recall some results about the generation of analytic semigroup in the spaces $L^{2}\left(\mathbb{R}^{d}\right)$ proved in [11] . In Section 3 we prove the generation of analytic semigroup and we obtain the domain characterization in the spaces $L^{p}\left(\mathbb{R}^{d}\right), 2<p<\infty$ and $L^{\infty}\left(\mathbb{R}^{d}\right)$. This result implies the generation of analytic semigroup in the spaces $L^{p}\left(\mathbb{R}^{d}\right)$ for $1 \leq p<2$ using duality techniques. 


\section{Preliminary material and notation}

Let $\Omega$ be an open subset of the $d$-dimensional Euclidean space $\mathbb{R}^{d}$. We denote by $C^{\infty}(\Omega)$ the linear space of all infinitely differentiable complex-valued functions on $\Omega$, and we write $C_{c}^{\infty}(\Omega)$ for the linear submanifold of $C^{\infty}(\Omega)$ of all functions with compact support in $\Omega$.

We denote by $W^{n, p}(\Omega)$ the usual Sobolev space (see, e.g., [1]), defined as the completion of $C_{c}^{\infty}(\Omega)$ with respect to the norm

$$
\|u\|_{W^{n, p}(\Omega)} \equiv \sum_{|\alpha| \leq n}\left(\int_{\Omega}\left|D^{\alpha} u(x)\right|^{p} d x\right)^{1 / p}
$$

writing $L^{p}(\Omega)\left[\right.$ resp. $\left.H^{n}(\Omega)\right]$ rather than $W^{0, p}(\Omega)\left[\right.$ resp. $\left.W^{n, 2}(\Omega)\right]$, and using the shorthands $W^{n, p}$ and $L^{p}$ for $W^{n, p}\left(\mathbb{R}^{d}\right)$ and $L^{p}\left(\mathbb{R}^{d}\right)$, respectively.

We denote by $W_{l o c}^{n, p}\left[\operatorname{resp} . L_{l o c}^{p}, H_{l o c}^{n}\right.$ ] the linear space of all measurable complexvalued functions on $\mathbb{R}^{d}$ belonging to $W^{n, p}(\Omega)\left[\right.$ resp. $\left.L^{p}(\Omega), H^{n}(\Omega)\right]$ for every open subset $\Omega$ of $\mathbb{R}^{d}$ having compact closure, and, for any fixed real-valued function $\xi \in W_{l o c}^{n, p}$, we define the weighted Sobolev space $W_{\xi}^{n, p}$ as the completion of $C_{c}^{\infty}\left(\mathbb{R}^{d}\right)$ with respect to the weighted norm

$$
\|u\|_{W_{\xi}^{n, p}} \equiv\|\xi u\|_{W^{n, p}}
$$

It is well known that $W_{\xi}^{n, p}$ can also be defined as the space of all measurable functions $u$ such that $\xi u \in W^{n, p}$. Similarly, for any choice of the functions $\alpha, \beta_{i}$, $i=1, \ldots, d, \delta_{i, j}, i, j=1, \ldots, d$ belonging to $L_{l o c}^{p}$, with $\operatorname{ssinf}|\alpha|>0$, we introduce the weighted Sobolev spaces $W_{(\alpha, \beta)}^{1, p}$ and $W_{(\alpha, \beta, \delta)}^{2, p}$ defined as the completion of $C_{c}^{\infty}\left(\mathbb{R}^{d}\right)$ with respect to the weighted norm

$$
\|u\|_{W_{(\alpha, \beta)}^{1, p}} \equiv\|\alpha u\|_{L^{p}}+\sum_{i=1}^{d}\left\|\beta_{i} D_{i} u\right\|_{L^{p}}
$$

and

$$
\|u\|_{W_{(\alpha, \beta, \delta)}^{2, p}} \equiv\|\alpha u\|_{L^{p}}+\sum_{i=1}^{d}\left\|\beta_{i} D_{i} u\right\|_{L^{p}}+\sum_{i, j=1}^{d}\left\|\delta_{i, j} D_{i, j} u\right\|_{L^{p}}
$$

respectively and we introduce also the spaces $W_{\xi,(\alpha, \beta)}^{1, p}\left[\operatorname{resp} . W_{\xi,(\alpha, \beta, \delta)}^{2, p}\right]$ of all measurable functions $u$ such that $\xi u \in W_{(\alpha, \beta)}^{1, p}\left[\right.$ resp. $\left.\xi u \in W_{(\alpha, \beta, \delta)}^{2, p}\right]$, endowed with the norms

$$
\|u\|_{W_{\xi,(\alpha, \beta)}^{1, p}} \equiv\|\xi u\|_{W_{(\alpha, \beta)}^{1, p}} \quad\left[\text { resp. }\|u\|_{W_{\xi,(\alpha, \beta, \delta)}^{2, p}} \equiv\|\xi u\|_{W_{(\alpha, \beta, \delta)}^{2, p}}\right] .
$$

Lastly we denote with $L_{\xi}^{p}$ the space $W_{\xi}^{0, p}$.

Let us now consider the formal second-order differential operator

$$
\mathcal{A} u \equiv \sum_{i, j=1}^{d} \psi_{i}(x) \psi_{j}(x) a_{i, j}(x) D_{i, j} u+\sum_{i=1}^{d} b_{i}(x) D_{i} u-\gamma^{2}(x) u
$$


Assumption 2.1. 1. For all $i, j=1, \ldots, d$, the coefficients $a_{i, j}(x)$ are bounded differentiable real-valued functions on $\mathbb{R}^{d}$ such that $a_{i, j}(x)=a_{j, i}(x)$, and satisfying the strong ellipticity condition

$$
R e \sum_{i, j=1}^{d} a_{i, j}(x) z_{i} \bar{z}_{j} \geq E|z|^{2} \quad \forall z \in \mathbb{C}^{d}
$$

for a suitable ellipticity modulus $E>0$ independent of $x \in \mathbb{R}^{d}$;

2 . for every $i=1, \ldots, d$, the coefficients $b_{i}(x)$ are measurable real-valued functions on $\mathbb{R}^{d}$, while $\gamma(x)$ is a real-valued function in $L_{l o c}^{2}$ with $\operatorname{essinf}(\gamma) \geq 1$;

3. for all $i=1, \ldots, d$ the coefficients $\psi_{i}(x)$ are differentiable, and we have

$$
\begin{gathered}
\left|b_{i}(x)\right| \leq B_{1} E^{1 / 2} \eta_{1, i}(x)\left|\psi_{i}(x)\right| \gamma(x) \quad \forall x \in \mathbb{R}^{d}, \\
\left|D_{j}\left(\psi_{i}(x) \psi_{j}(x) a_{i, j}(x)\right)\right| \leq B_{2} E^{1 / 2} \eta_{2, i, j}(x)\left|\psi_{i}(x)\right| \gamma(x) \quad \forall x \in \mathbb{R}^{d},
\end{gathered}
$$

for suitable constants $B_{1}$ and $B_{2}$ such that $B_{1}+B_{2}<2$ and measurable positive functions $\eta_{1, i}(x)$ and $\eta_{2, i, j}(x)$ satisfying

$$
\sum_{i=1}^{d} \eta_{1, i}^{2}(x)=d \sum_{i, j=1}^{d} \eta_{2, i, j}^{2}(x)=1
$$

Assumption 2.1 allows us to reduce the analysis of the nonvariational case to the analysis of the variational one. Indeed, introducing the sesquilinear form $a(\cdot, \cdot)$ associated to the operator $\mathcal{A}$, given by

$$
a(u, v) \equiv \widehat{a}(u, v)-\int_{\mathbb{R}^{d}} \sum_{i, j=1}^{d} D_{j}\left(\psi_{i}(x) \psi_{j}(x) a_{i, j}(x)\right) D_{i} u(x) \bar{v}(x) d x,
$$

for all $u \in H_{(\gamma, \psi)}^{1, p} 1<p<\infty$ and $v \in H_{(\gamma, \psi)}^{1, q}$ where $q$ is the conjugate of $p$, and writing

$$
D\left(\mathcal{A}_{p}\right) \equiv\left\{u \in H_{(\gamma, \psi)}^{1, p}: \exists K(u)>0 \text { s.t. }|a(u, \varphi)| \leq K(u)\|\varphi\|_{q} \forall \varphi \in C_{c}^{\infty}\left(\mathbb{R}^{d}\right)\right\},
$$

one can study the realization $\mathcal{A}_{p}: D\left(\mathcal{A}_{p}\right) \rightarrow L^{p}$ of $\mathcal{A}$ by considering for each $\lambda \in \mathbb{C}$ such that $\operatorname{Re} \lambda>0$, the equation

$$
\left(\lambda-\mathcal{A}_{p}\right) u=f .
$$

In [11] the following results are proved:

Theorem 2.2. Under Assumption 2.1, the operator $\mathcal{A}_{2}: D\left(\mathcal{A}_{2}\right) \rightarrow L^{2}$ generates an analytic semigroup on $L^{2}$.

Moreover

\footnotetext{
${ }^{1}$ This condition could be replaced by the seemingly more general essinf $\gamma>0$, provided to employ a standard normalization procedure.
} 
Corollary 2.3. Under Assumption 2.1, for every solution $u \in D\left(\mathcal{A}_{2}\right)$ of $(3)$, we have

$$
|\lambda|^{1 / 2}\|\gamma u\|_{L^{2}} \leq K^{\prime}\|f\|_{L^{2}} \quad \text { and } \quad|\lambda|^{1 / 2}\left\|\psi_{i} D_{i} u\right\|_{L^{2}} \leq K^{\prime \prime}\|f\|_{L^{2}}
$$

for suitably chosen $K^{\prime}, K^{\prime \prime}>0$ independent of $\lambda$.

In order to obtain suitable estimates for the first-order derivatives we need the following assumption:

Assumption 2.4. Under 1. and 2. of Assumption 2.1, suppose in addition that $\gamma$ is continuously differentiable and that, for all $i, j=1, \ldots, d$ and $x \in \mathbb{R}^{d}$, we have

$$
\begin{gathered}
\left|b_{i}(x)\right| \leq B_{1} E^{1 / 2} \eta_{1, i}(x)\left|\psi_{i}(x)\right| \gamma(x), \\
\left|D_{j}\left(\psi_{i}(x) \psi_{j}(x) a_{i, j}(x)\right)\right| \leq B_{2} E^{1 / 2} \eta_{2, i, j}(x)\left|\psi_{i}(x)\right| \gamma(x), \\
2\left|\psi_{j}(x) D_{j} \gamma(x) a_{i, j}(x)\right| \leq B_{3} E^{1 / 2} \eta_{3, i, j}(x) \gamma^{2}(x),
\end{gathered}
$$

for suitable constants $B_{1}, B_{2}$ and $B_{3}$ such that $B_{1}+B_{2}+B_{3}<2$ and suitable measurable functions $\eta_{1, i}(x), \eta_{2, i, j}(x)$ and $\eta_{3, i, j}(x)$ on $\mathbb{R}^{d}$ satisfying $\sum_{i=1}^{d} \eta_{1, i}(x)=$ $d \sum_{i, j=1}^{d} \eta_{2, i, j}^{2}(x)=d \sum_{i, j=1}^{d} \eta_{3, i, j}^{2}(x)=1$.

We have

Theorem 2.5. Under Assumption 2.4, both $\gamma^{2} u$ and $\psi_{i} \gamma D_{i} u$ belong to $L^{2}$, for every $i=1, \ldots, d$. More precisely, $u$ belongs to $H_{\left(\gamma^{2}, \gamma \psi\right)}^{1}$, and

$$
\|u\|_{H_{\left(\gamma^{2}, \gamma \psi\right)}^{1}} \leq K\|f\|_{L^{2}}
$$

holds true for a suitable $K>0$. In particular, for every $i=1, \ldots, d$, also $b_{i} D_{i} u$ belongs to $L^{2}$, and we have

$$
\sum_{i=1}^{d}\left\|b_{i} D_{i} u\right\|_{L^{2}} \leq d^{2} B_{1} E^{1 / 2} \sum_{i=1}^{d}\left\|\psi_{i} \gamma D_{i} u\right\|_{L^{2}} .
$$

Aiming to show that for all $i, j=1, \ldots, d$ the single summand $\psi_{i}(x) \psi_{j}(x) D_{i, j} u(x)$ belongs to $L^{2}$, we need to strengthen our hypotheses on the coefficients $\psi_{i}(x)$ 's. Therefore, having in mind our examples, we will assume then the negligibility of the set

$$
Z \equiv\left\{x \in \mathbb{R}^{d}: \psi_{i}(x)=0, \text { for some } i=1, \ldots, d\right\},
$$

of all zeros of the $\psi_{i}(x)$ 's, and the existence of a suitable countable covering of $\mathbb{R}^{d}-Z$ which allows us to perform a localization procedure. Such a covering will be made by rectangles of the type

$$
R\left(x_{0}, r \psi\right) \equiv\left\{x \in \mathbb{R}^{d}:\left|x_{i}-x_{i}^{(0)}\right| \leq r\left|\psi_{i}\left(x_{0}\right)\right|, i=1, \ldots, d\right\},
$$

for $x_{0} \equiv\left(x_{1}^{(0)}, \ldots, x_{d}^{(0)}\right) \in \mathbb{R}^{d}-Z$ and $r>0$.

Assumption 2.6. Under Assumption 2.4, suppose in addition that 
(i) for every $i=1, \ldots, d$, the differentiable function $\psi_{i}(x)$ belongs to $H_{l o c}^{1}$ and the set $Z$ is negligible;

(ii) there exist real numbers $r_{1}>0$ and $L>0$ such that for every $0<r \leq r_{1}$ we can find a countable set $N_{r} \subset \mathbb{R}^{d}-Z$ such that

(a) the family $\mathcal{F}_{1} \equiv\{R(x, r \psi)\}_{x \in N_{r}}$ is a covering of $\mathbb{R}^{d}-Z$;

(b) each rectangle of the family $\mathcal{F}_{2} \equiv\{R(x, 2 r \psi)\}_{x \in N_{r}}$ does not contain any element of $Z$ and has a nonempty intersection with at most a fixed number $n_{0}$ of other rectangles of $\mathcal{F}_{2}$ itself;

(c) we have

$$
\frac{1}{L} \leq \min _{i=1, \ldots, d} \inf _{x \in R\left(x_{0}, 2 r \psi\right)} \frac{\left|\psi_{i}(x)\right|}{\left|\psi_{i}\left(x_{0}\right)\right|} \leq \max _{i=1, \ldots, d} \sup _{x \in R\left(x_{0}, 2 r \psi\right)} \frac{\left|\psi_{i}(x)\right|}{\left|\psi_{i}\left(x_{0}\right)\right|} \leq L,
$$

for each $x_{0} \in N_{r}$

Remark 2.7. Assumption 2.6 is convenient for proving the characterization result. However, it is not easy to check it for given operators. In [11] it is proved that 2.6 is verified under a more treatable assumption, befitted with examples coming from financial mathematics. More precisely, it was shown that the Assumption 2.8 below implies 2.6.

Assumption 2.8. Under Assumption 2.4, suppose in addition that

(i) Part (i) of Assumption 2.6 holds true;

(ii) there exist $r_{0}>0$ (small), $R_{0}>0$ (large), and $\alpha>0$ such that for every $x \in\left\{x \in \mathbb{R}^{d}: \operatorname{dist}(x, Z)<r_{0}\right.$ or $\left.\operatorname{dist}(x, 0)>R_{0}\right\} \equiv \mathcal{D}\left(r_{0}, R_{0}\right)$ and every $i=$ $1, \ldots, d$ we have

$$
\left|D_{j} \psi_{i}(x)\right| \leq \alpha
$$

(iii) for every $i=1, \ldots, d$ the function $\psi_{i}(x)$ depends only on the variable $x_{i}$.

Theorem 2.9. Under Assumption 2.6, the functions

$$
\psi_{i}(x) \psi_{j}(x) D_{i, j} u(x)
$$

belong to $L^{2}$ for all $i, j=1, \ldots, d$. More precisely, we have $u \in H_{\left(\gamma^{2}, \gamma \psi, \psi^{2}\right)}^{2}$ and the estimate

$$
|\lambda|\|u\|_{L^{2}}+|\lambda|^{1 / 2}\|u\|_{H_{(\gamma, \psi)}^{1}}+\|u\|_{H_{\left(\gamma^{2}, \gamma \psi, \psi^{2}\right)}^{2}} \leq K\|f\|_{L^{2}}
$$

holds true for a suitable $K>0$.

Such results can be extended to the case of weighted Sobolev spaces:

Theorem 2.10. Assume that Assumption 2.4 still holds true when replacing the first-order term of the operator $\mathcal{A}$ with

$$
\sum_{i=1}^{d} b_{i} D_{i}+\sum_{i, j=1}^{d} \psi_{i} \psi_{j} a_{i, j}\left(\frac{D_{i} \xi}{\xi} D_{j}+\frac{D_{j} \xi}{\xi} D_{i}\right)
$$


and the zero-order term with

$$
-\gamma^{2}+\sum_{i, j=1}^{d} \psi_{i} \psi_{j} a_{i, j}\left(\frac{D_{i, j} \xi}{\xi}+2 \frac{D_{i} \xi D_{j} \xi}{\xi^{2}}\right)+\sum_{i=1}^{d} b_{i} \frac{D_{i} \xi}{\xi}
$$

then the operator $\mathcal{A}$ has a realization $\mathcal{A}_{2, \xi}: D\left(\mathcal{A}_{2, \xi}\right) \rightarrow L_{\xi}^{2}$ which generates an analytic semigroup on $L_{\xi}^{2}$. Moreover, for each $\lambda \in \mathbb{C}$ such that $\operatorname{Re} \lambda>0$, the resolvent equation $\lambda u-\mathcal{A}_{2, \xi} u=f$ has, for every $f \in L^{2}$, a unique solution $u \in$ $D\left(\mathcal{A}_{2, \xi}\right)$, which satisfies the estimate

$$
|\lambda|\|u\|_{L_{\xi}^{2}}+|\lambda|^{1 / 2}\|u\|_{H_{\xi,(\gamma, \psi)}^{1,2}}+\|u\|_{H_{\xi,\left(\gamma^{2}, \gamma \psi, \psi^{2}\right)}^{2,2}} \leq C\|f\|_{L_{\xi}^{2}}
$$

for a suitable constant $C>0$. In particular we have $D\left(\mathcal{A}_{2, \xi}\right)=H_{\xi,\left(\gamma^{2}, \gamma \psi, \psi^{2}\right)}^{2}$.

Remark 2.11. By using the Korn's argument it is possible to pass from generation results in the case of differentiable coefficients to similar result in the case of continuous coefficients. However, in such a general setting, it is impossible to find a general approach leading to this kind of results. This can be done in particular cases with different procedures.

\section{Generation of analytic semigroups on $L^{p}\left(\mathbb{R}^{d}\right)$}

In order to prove the $L^{p}$ estimates we need an additional assumption. Actually we need the existence of a suitable countable covering of $\mathbb{R}^{d}-Z$ which allows us to perform a localization procedure. Let $r_{\gamma\left(x_{0}\right)}$ be the minimum between $r$ and $\gamma\left(x_{0}\right)^{-1}$. Let

$$
R\left(x_{0}, r_{\psi, \gamma}\right) \equiv\left\{x \in \mathbb{R}^{d}:\left|x_{i}-x_{i}^{(0)}\right| \leq r_{\gamma\left(x_{0}\right)}\left|\psi_{i}\left(x_{0}\right)\right|, i=1, \ldots, d\right\},
$$

for $x_{0} \equiv\left(x_{1}^{(0)}, \ldots, x_{d}^{(0)}\right) \in \mathbb{R}^{d}-Z$ and $r>0$.

Assumption 3.1. Under Assumption 2.4, suppose in addition that

(i) Part (i) of Assumption 2.6 holds true;

(ii) There exist real numbers $r_{1}>0$ and $L>0$ such that for every $0<r \leq r_{1}$ we can find a countable set $N_{r} \subset \mathbb{R}^{d}-Z$ such that

(a) the family $\mathcal{F}_{1} \equiv\left\{R\left(x, r_{\psi, \gamma}\right)\right\}_{x \in N_{r}}$ is a covering of $\mathbb{R}^{d}-Z$;

(b) each rectangle of the family $\mathcal{F}_{2} \equiv\left\{R\left(x, 2 r_{\psi, \gamma}\right)\right\}_{x \in N_{r}}$ does not contain any element of $Z$ and has a nonempty intersection with at most a fixed number $n_{0}$ of other rectangles of $\mathcal{F}_{2}$ itself;

(c) we have

$$
\frac{1}{L} \leq \inf _{x \in R\left(x_{0}, 2 r_{\psi, \gamma}\right)} \frac{|\gamma(x)|}{\left|\gamma\left(x_{0}\right)\right|} \leq \sup _{x \in R\left(x_{0}, 2 r_{\psi, \gamma}\right)} \frac{|\gamma(x)|}{\left|\gamma\left(x_{0}\right)\right|} \leq L,
$$

for each $x_{0} \in N_{r}$. 
Remark 3.2. As done in Remark 2.7, it is possible to find stronger conditions that imply Assumption 3.1 and that are usually satisfied by the classical problems arising from financial mathematics.

Lemma 3.3. Under Assumption 3.1, assume to have proved that for a $p \geq 2$ the solution $u$ of the resolvent equation (3), related to some $\lambda \in \mathbb{C}$ such that $\operatorname{Re} \lambda>0$ belongs to $W_{\left(\gamma^{2}, \gamma \psi, \psi^{2}\right)}^{2, p}$ and satisfies the estimate

$$
|\lambda|\|u\|_{L^{p}}+|\lambda|^{1 / 2}\|u\|_{W_{(\gamma, \psi)}^{1, p}}+\|u\|_{W_{\left(\gamma^{2}, \gamma \psi, \psi^{2}\right)}^{2, p}} \leq C^{\prime}\|f\|_{L^{p}}
$$

for some $C^{\prime}>0$ and for each $f \in L^{p}$.

Let $q \in\left(p, p^{*}\right)$ and let $f \in L^{q}$. Assume that $u \in W_{\left(\gamma^{2}, \gamma \psi\right)}^{1, p}$ is a solution of (3). Then $u$ satisfies the estimate

$$
|\lambda|\|u\|_{L^{q}}+|\lambda|^{1 / 2}\|u\|_{W_{(\gamma, \psi)}^{1, q}}+\|u\|_{W_{\left(\gamma^{2}, \gamma \psi\right)}^{1, q}} \leq C\|f\|_{L^{q}}
$$

for every $\lambda \in \mathbb{C}$ whose real part is greater than a suitable fixed positive real number $\omega$ and for a suitable $C>0$ independent of $\lambda$. Moreover if $p>d$ we may choose $q=\infty$.

Proof. For each $x_{0} \equiv\left(x_{1}^{0}, \ldots, x_{d}^{0}\right) \in \mathbb{R}^{d}-Z$, we also consider the change of variables $T_{x_{0}, \psi}: \mathbb{R}^{d} \mapsto \mathbb{R}^{d}$ defined by

$$
T_{x_{0}, \psi}(x) \stackrel{\text { def }}{=}\left(\left(x_{1}-x_{1}^{0}\right) /\left|\psi_{1}\left(x_{0}\right)\right|, \ldots,\left(x_{d}-x_{d}^{0}\right) /\left|\psi_{d}\left(x_{0}\right)\right|\right),
$$

of inverse

$$
T_{x_{0}, \psi}^{-1}(x)=\left(x_{1}^{0}+\left|\psi_{1}\left(x_{0}\right)\right| x_{1}, \ldots, x_{d}^{0}+\left|\psi_{d}\left(x_{0}\right)\right| x_{d}\right) .
$$

Furthermore, for every $r>0$ we denote by $B\left(x_{0}, r\right)$ the $d$-dimensional ball centered at 0 with radius $r$ and we write $B\left(x_{0}, r \psi\right)$ for the $d$-dimensional ellipsoid centered at $x_{0}$ with semiaxes $r\left|\psi_{1}\left(x_{0}\right)\right|, \ldots, r\left|\psi_{d}\left(x_{0}\right)\right|$. Clearly

$$
T_{x_{0}, \psi}\left(B\left(x_{0}, r \psi\right)\right)=B(0, r) \quad \text { and } \quad T_{x_{0}, \psi}^{-1}(B(0, r))=B\left(x_{0}, r \psi\right) .
$$

Consider the change of variables

$$
\widetilde{u}(x) \stackrel{\text { def }}{=}\left(u \circ T_{x_{0}, \psi}^{-1}\right)(x),
$$

and let $\theta(x)$ be any smooth cut-off function such that

$$
\left\{\begin{array}{ll}
\theta(t)=1 & \text { if } t \in[0,1] \\
\theta(t)=0 & \text { if } t \in[2,+\infty[
\end{array},\right.
$$

for each $0<r \leq r_{0}$ we can define a cut-off function on $\mathbb{R}^{d}$ by setting

$$
\theta_{r}(x) \stackrel{\text { def }}{=} \theta\left(\frac{|x|}{r}\right)
$$

and we can consider the function

$$
v(x)=\theta_{r}(x) \widetilde{u}(x) .
$$


Clearly $v \in L^{p}$ satisfies the following equation

$$
\begin{aligned}
& \sum_{i, j=1}^{d} \frac{\widetilde{\psi}_{i}(x) \widetilde{\psi}_{j}(x)}{\psi_{i}\left(x_{0}\right) \psi_{j}\left(x_{0}\right)} \widetilde{a}_{i, j}(x) D_{i, j} v(x)+\sum_{i=1}^{d} \frac{\widetilde{b}_{i}(x)}{\psi_{i}\left(x_{0}\right)} D_{i} v(x)-\widetilde{\gamma}^{2} v(x)-\lambda v(x) \\
& \quad=\theta_{r}(x) \widetilde{f}(x)+\sum_{i=1}^{d} \frac{\widetilde{b}_{i}(x)}{\psi_{i}\left(x_{0}\right)} D_{i} \theta_{r}(x) \widetilde{u}(x) \\
& \quad+\sum_{i, j=1}^{d} \frac{\widetilde{\psi}_{i}(x) \widetilde{\psi}_{j}(x)}{\psi_{i}\left(x_{0}\right) \psi_{j}\left(x_{0}\right)} \widetilde{a}_{i, j}(x)\left[\widetilde{u}(x) D_{i, j} \theta_{r}(x)+D_{i} \theta_{r}(x) D_{j} \widetilde{u}(x)+D_{j} \theta_{r}(x) D_{i} \widetilde{u}(x)\right]
\end{aligned}
$$

whose right side $\tilde{h}$ satisfies

$$
\widetilde{h} \in L^{p}(B(0,2 r)) \quad \text { and } \quad \widetilde{h}=0 \text { on } \partial B(0,2 r) .
$$

On the other hand, by the assumption of the lemma the solution $v \in L^{p}$ satisfies the estimate

$$
|\lambda|\|v\|_{L^{p}}+|\lambda|^{1 / 2}\|v\|_{W_{(\gamma, \psi)}^{1, p}}+\|v\|_{W_{\left(\gamma, \psi \gamma, \psi^{2}\right)}^{2, p}} \leq C_{1}\|\widetilde{h}\|_{L^{p}}
$$

for a suitable $C>0$ independent of $\lambda$.

Furthermore we can also prove that

$$
\begin{aligned}
\|\widetilde{h}\|_{L^{p}} \leq & C_{2}\left[\|\widetilde{f}\|_{L^{p}(B(0,2 r))}+\frac{1}{r^{2}}\|\widetilde{u}\|_{L^{p}(B(0,2 r))}+\frac{1}{r}\|D \widetilde{u}\|_{L^{p}(B(0,2 r))}\right. \\
& \left.+\frac{1}{r}\|\widetilde{\gamma} \widetilde{u}\|_{L^{p}(B(0,2))}\right]
\end{aligned}
$$

for a suitable $C_{2}>0$ where the last estimate comes from equation (2).

Combining the above estimates, we obtain

$$
\begin{aligned}
& |\lambda|\|\widetilde{u}\|_{L^{p}(B(0, r))}+|\lambda|^{1 / 2}\left[\gamma\left(x_{0}\right)\|\widetilde{u}\|_{L^{p}(B(0, r))}+\|D \widetilde{u}\|_{L^{p}(B(0, r))}\right] \\
& +\gamma^{2}\left(x_{0}\right)\|\widetilde{u}\|_{L^{p}(B(0, r))}+\gamma\left(x_{0}\right)\|D \widetilde{u}\|_{L^{p}(B(0, r))}+\left\|D^{2} \widetilde{u}\right\|_{L^{p}(B(0, r))} \\
& \leq C\left[\|\widetilde{f}\|_{L^{p}(B(0,2 r))}+\frac{1}{r^{2}}\|\widetilde{u}\|_{L^{p}(B(0,2 r))}+\frac{1}{r}\|D \widetilde{u}\|_{L^{p}(B(0,2 r))}\right. \\
& \left.\quad+\frac{1}{r} \gamma\left(x_{0}\right)\|\widetilde{u}\|_{L^{p}(B(0,2 r))}\right],
\end{aligned}
$$

where we suppose $r$ small enough in order that Assumption 3.1 holds. Now, if we take $q \in\left(p, p^{*}\right)$ and $\delta(q)=d / q-d / p+1$, for every $\varepsilon>0$ there exists (see [13, p. 66]) $C(\varepsilon)>0$ such that

$$
\|\widetilde{u}\|_{L^{q}(B(0, r))} \leq \varepsilon r^{\delta(q)}\|D \widetilde{u}\|_{L^{p}(B(0, r))}+C(\varepsilon) r^{\delta(q)-1}\|\widetilde{u}\|_{L^{p}(B(0, r))},
$$

and

$$
\|D \widetilde{u}\|_{L^{q}(B(0, r))} \leq \varepsilon r^{\delta(q)}\left\|D^{2} \widetilde{u}\right\|_{L^{p}(B(0, r))}+C(\varepsilon) r^{\delta(q)-2}\|\widetilde{u}\|_{L^{p}(B(0, r))} .
$$


Combining estimates (7) and (8) we have

$$
\begin{aligned}
& \frac{1}{r^{2}}\|\widetilde{u}\|_{L^{q}(B(0, r))}+\frac{1}{r}\|D \widetilde{u}\|_{L^{q}(B(0, r))} \\
& \leq \quad \frac{1}{r^{2}}\left[\varepsilon r^{\delta(q)}\|D \widetilde{u}\|_{L^{p}(B(0, r))}+C(\varepsilon) r^{\delta(q)-1}\|\widetilde{u}\|_{L^{p}(B(0, r))}\right] \\
& \quad+\frac{1}{r}\left[\varepsilon r^{\delta(q)}\left\|D^{2} \widetilde{u}\right\|_{L^{p}(B(0, r))}+C(\varepsilon) r^{\delta(q)-2}\|\widetilde{u}\|_{L^{p}(B(0, r))}\right] .
\end{aligned}
$$

and, rearranging the terms,

$$
\begin{aligned}
& \frac{1}{r^{2}}\|\widetilde{u}\|_{L^{q}(B(0, r))}+\frac{1}{r}\|D \widetilde{u}\|_{L^{q}(B(0, r))} \\
& \leq C(\varepsilon) r^{\delta(q)-3}\|\widetilde{u}\|_{L^{p}(B(0, r))}+\varepsilon r^{\delta(q)-2}\|D \widetilde{u}\|_{L^{p}(B(0, r))}+\varepsilon r^{\delta(q)-1}\left\|D^{2} \widetilde{u}\right\|_{L^{p}(B(0, r))} .
\end{aligned}
$$

Taking into account (6), the above estimate implies

$$
\begin{aligned}
& \frac{1}{r^{2}}\|\widetilde{u}\|_{L^{q}(B(0, r))}+\frac{1}{r}\|D \widetilde{u}\|_{L^{q}(B(0, r))} \\
& \leq\left[C(\varepsilon) r^{\delta(q)-3}\left(|\lambda|+\gamma\left(x_{0}\right)^{2}\right)^{-1}+\varepsilon r^{\delta(q)-2}\left(|\lambda|^{\frac{1}{2}}+\gamma\left(x_{0}\right)\right)^{-1}+\varepsilon r^{\delta(q)-1}\right] \\
& {\left[\|\widetilde{f}\|_{L^{p}(B(0,2 r))}+\frac{1}{r^{2}}\|\widetilde{u}\|_{L^{p}(B(0,2 r))}+\frac{1}{r}\|D \widetilde{u}\|_{L^{p}(B(0,2 r))}+\frac{1}{r} \gamma\left(x_{0}\right)\|\widetilde{u}\|_{L^{p}(B(0,2 r))}\right] .}
\end{aligned}
$$

By Hoelder inequality we get

$$
\begin{aligned}
& \frac{1}{r^{2}}\|\widetilde{u}\|_{L^{q}(B(0, r))}+\frac{1}{r}\|D \widetilde{u}\|_{L^{q}(B(0, r))} \\
& \leq\left[C(\varepsilon) r^{-2}\left(|\lambda|+\gamma\left(x_{0}\right)^{2}\right)^{-1}+\varepsilon r^{-1}\left(|\lambda|^{\frac{1}{2}}+\gamma\left(x_{0}\right)\right)^{-1}+\varepsilon\right] \\
& {\left[\|\widetilde{f}\|_{L^{q}(B(0,2 r))}+\frac{1}{r^{2}}\|\widetilde{u}\|_{L^{q}(B(0,2 r))}+\frac{1}{r}\|D \widetilde{u}\|_{L^{q}(B(0,2 r))}+\frac{1}{r} \gamma\left(x_{0}\right)\|\widetilde{u}\|_{L^{q}(B(0,2 r))}\right] .}
\end{aligned}
$$

Finally, if we take:

- $\varepsilon>0$ a small number to be chosen later

- $r_{0}=\left(|\lambda|^{\frac{1}{2}}+\gamma\left(x_{0}\right)\right)^{-1}$

- $r=\alpha r_{0}$, where $\alpha$ is a number to be chosen later,

then we obtain

$$
\begin{aligned}
& \frac{1}{\alpha^{2}}\left(|\lambda|^{\frac{1}{2}}+\gamma\left(x_{0}\right)\right)^{2}\|\widetilde{u}\|_{L^{q}\left(B\left(0, r_{0}\right)\right)}+\frac{1}{\alpha}\left(|\lambda|^{\frac{1}{2}}+\gamma\left(x_{0}\right)\right)\|D \widetilde{u}\|_{L^{q}\left(B\left(0, r_{0}\right)\right)} \\
& \leq\left[C(\varepsilon) \frac{1}{\alpha^{2}}+\varepsilon \frac{1}{\alpha}+\varepsilon\right]\left[\|\widetilde{f}\|_{L^{q}\left(B\left(0,2 r_{0}\right)\right)}+\frac{1}{\alpha^{2}}\left(|\lambda|^{\frac{1}{2}}+\gamma\left(x_{0}\right)\right)^{2}\|\widetilde{u}\|_{L^{q}\left(B\left(0,2 r_{0}\right)\right)}\right. \\
& \left.+\frac{1}{\alpha}\left(|\lambda|^{\frac{1}{2}}+\gamma\left(x_{0}\right)\right)\|D \widetilde{u}\|_{L^{q}\left(B\left(0,2 r_{0}\right)\right)}+\frac{1}{\alpha}\left(|\lambda|^{\frac{1}{2}}+\gamma\left(x_{0}\right)\right) \gamma\left(x_{0}\right)\|\widetilde{u}\|_{L^{q}\left(B\left(0,2 r_{0}\right)\right)}\right]
\end{aligned}
$$


So if $q<\infty$, by changing variable back, summing up to the covering and using assumption 3.1 , we have

$$
\begin{aligned}
& \frac{1}{\alpha^{2}}\left(|\lambda|\|u\|_{L^{q}}+\left\|\gamma^{2} u\right\|_{L^{q}}+\frac{1}{\alpha}\left(|\lambda|^{1 / 2}\|D u\|_{L^{q}}+\|\gamma D u\|_{L^{q}}\right)\right. \\
& \leq \quad 2 L^{4} n_{0}\left[C(\varepsilon) \frac{1}{\alpha^{2}}+\varepsilon \frac{1}{\alpha}+\varepsilon\right]\left[\frac{1}{\alpha^{2}}\left(|\lambda|\|u\|_{L^{q}}+\left\|\gamma^{2} u\right\|_{L^{q}}\right)\right. \\
& \left.\quad+\frac{1}{\alpha}\left(|\lambda|^{1 / 2}\|\gamma u\|_{L^{q}}+\left\|\gamma^{2} u\right\|_{L^{q}}\right)+\frac{1}{\alpha}\left(|\lambda|^{1 / 2}\|D u\|_{L^{q}}+\|\gamma D u\|_{L^{q}}\right)+\|f\|_{L^{q}}\right] .
\end{aligned}
$$

The statement follows from the above estimate choosing $\varepsilon=\frac{1}{8 L^{4} n_{0}}$ and $\alpha=$ $4 C(\varepsilon) L^{4} n_{0}$. If $q=\infty$ the argument is easier. Actually by changing variable back and by localizing around the points where $u(x), \gamma^{2}(x) u(x), D u(x), \psi(x) D u(x)$ and $\gamma(x) \psi(x) D u(x)$ attain the maximum, the result follows directly from (9) without using a covering argument.

Remark 3.4. Assume $f \in L^{\infty}$. Arguing as in [15] from the proof of the previous lemma it is possible to get an estimate for the second derivatives. Actually, starting from equation (6), by Hoelder inequality and choosing a suitable $r=\alpha\left(|\lambda|^{\frac{1}{2}}+\right.$ $\left.\gamma\left(x_{0}\right)\right)^{-1}$, one gets that for each $q>d$ :

$$
\sup _{x_{0} \in \mathbb{R}^{d}}\left(|\lambda|^{\frac{1}{2}}+\gamma\left(x_{0}\right)\right)^{\frac{d}{q}}\left\|\psi^{2} D^{2} u\right\|_{L^{q}\left(B\left(x_{0}, r_{0} \psi\right)\right)} \leq C\|f\|_{L^{\infty}} .
$$

Remark 3.5. Estimate (4) implies the uniqueness of the solution of (3) in $W_{\gamma^{2}, \gamma \psi}^{1, q}$.

Lemma 3.6. Under the assumptions of Lemma 3.3 we have that $u \in W_{\gamma^{2}, \gamma \psi, \psi^{2}}^{2, q}$ and

$$
\left\|\psi^{2} D^{2} u\right\|_{L^{q}} \leq C\|f\|_{L^{q}}
$$

Proof. Using the notation of the previous lemma we have that the function $\widetilde{u}(x)$ satisfies the following equation in $B\left(x_{0}, 2 r\right)$

$$
\left.\sum_{i, j=1}^{d} \frac{\widetilde{\psi}_{i}(x) \widetilde{\psi}_{j}(x)}{\psi_{i}\left(x_{0}\right) \psi_{j}\left(x_{0}\right)} \widetilde{a}_{i, j}(x) D_{i, j} \widetilde{u}(x)\right)=-\sum_{i=1}^{d} \frac{\widetilde{b}_{i}(x)}{\psi_{i}\left(x_{0}\right)} D_{i} \widetilde{u}(x)-\widetilde{\gamma}^{2} \widetilde{u}(x)+\widetilde{f}(x)=\widetilde{h}(x) .
$$

Noting that the second order differential operator

$$
\widetilde{u}(x) \mapsto \sum_{i, j=1}^{d} \frac{\widetilde{\psi}_{i}(x) \widetilde{\psi}_{j}(x)}{\psi_{i}\left(x_{0}\right) \psi_{j}\left(x_{0}\right)} \widetilde{a}_{i, j}(x) D_{i, j} \widetilde{u}(x)
$$

is a strongly elliptic operator in $B(0,2 r)$ and, thanks to known regularity results (see [6] and also [9, Theor. 17.2, p.67], [10, 8.3, p.173]) it follows that $\widetilde{u}(x) \in$ $W^{2, q}(B(0, r))$ and

$$
\left\|D^{2} \widetilde{u}\right\|_{L^{q}\left(B\left(x_{0}, 2 r\right)\right.} \leq C\|\widetilde{h}\|_{L^{q}\left(B\left(x_{0}, 2 r\right)\right.} .
$$

The statement follows changing variable back, summing up to the covering and applying the results of Lemma 3.3. 
Theorem 3.7. Assume that 3.1 holds. Then for each $2 \leq q \leq \infty$ the operator $\mathcal{A}_{q}: D\left(\mathcal{A}_{q}\right) \rightarrow L^{q}$ generates an analytic semigroup on $L^{p}$. Moreover if $q<\infty$ for every solution $u \in D\left(\mathcal{A}_{q}\right)$ of $(3)$, we have

$$
|\lambda|\|u\|_{L^{q}}+|\lambda|^{\frac{1}{2}}\|u\|_{W_{(\gamma, \psi)}^{1, q}}+\|u\|_{W_{\left(\gamma^{2}, \gamma \psi, \psi^{2}\right)}^{2, q}} \leq C\|f\|_{L^{q}}
$$

for every $\lambda \in \mathbb{C}$ whose real part is greater than a suitable fixed positive real number $\omega$ and for a suitable $C>0$ independent of $\lambda$.

If $q=\infty$ then for every solution $u \in D\left(\mathcal{A}_{\infty}\right)$ of $(3)$, we have

$$
|\lambda|\|u\|_{L^{\infty}}+|\lambda|^{\frac{1}{2}}\|u\|_{W_{(\gamma, \psi)}^{1, \infty}}+\|u\|_{W_{\left(\gamma^{2}, \gamma \psi\right)}^{1, \infty}} \leq C\|f\|_{L^{\infty}}
$$

for every $\lambda \in \mathbb{C}$ whose real part is greater than a suitable fixed positive real number $\omega$ and for a suitable $C>0$ independent of $\lambda$.

Proof. This Theorem is true for $p=2$. Let $q \in\left(2,2^{*}\right]$ where $2^{*}$ is the Sobolev exponent. Let $u$ be a solution of (3) and assume that $f \in L^{2} \cap L^{q}$. By applying Lemmata 3.3 and 3.6 we have that (11) holds.

If $f \in L^{q}$, we consider a sequence of functions $f_{n} \in L^{2} \cap L^{q}$ converging to $f$ in $L^{q}$. Then the sequence of associated solutions $u_{n}$ is a Cauchy sequence in $W_{\left(\gamma^{2}, \gamma \psi, \psi^{2}\right)}^{2, q}$ converging to a function $u$ that is a solution of (3). Moreover this solution is unique by Remark 3.5. Therefore the result is proved for any $q \in\left(2,2^{*}\right]$.

If $q \in\left(2^{*},\left(2^{*}\right)^{*}\right)$ one can prove the result iterating the previous argument. After a finite number of steps the statement follows.

Using duality techniques one may prove a generation result in $L^{p}$ with $1 \leq p<2$.

Theorem 3.8. Assume 3.1 holds. Let $1 \leq p<2$, then the operator $\widehat{A}_{p}: D\left(\mathcal{A}_{p}\right) \rightarrow L^{p}$ generates an analytic semigroup on $L^{p}$.

Proof. The statement follows if we show that a solution of (3) for $1 \leq p<2$ exists and is unique, and moreover

$$
|\lambda|\|u\|_{L^{p}} \leq C\|f\|_{L^{p}}
$$

for every $\lambda \in \mathbb{C}$ whose real part is greater than a suitable fixed positive real number $\omega$ and for a suitable $C>0$ independent of $\lambda$.

Assume first $f \in L^{p} \cap L^{2}$ so one has the existence of a solution of (3). Let $\widehat{\mathcal{A}}$ the operator in variational form

$$
\widehat{\mathcal{A}} u \equiv \sum_{i, j=1}^{d} D_{j}\left(\psi_{i}(x) \psi_{j}(x) a_{i, j}(x) D_{i} u\right)+\sum_{i=1}^{d} b_{i}(x) D_{i} u-\gamma^{2}(x) u,
$$

and let $\widehat{\mathcal{A}}^{*}$ its adjoint.

Define the function space $H=\left\{g \in L^{p^{\prime}}:\|g\|_{L^{p^{\prime}}}=1\right\}$ where $p^{\prime}$ is the conjugate of p. Then

$$
\|u\|_{L^{p}}=\sup _{g \in H} \int u g d x
$$


For each $g \in H$, let $v_{g}$ be the solution of the equation

$$
\left(\widehat{\mathcal{A}}^{*}-\lambda\right) v_{g}=g \text {. }
$$

By Theorem 3.7

$$
|\lambda|\left\|v_{g}\right\|_{L^{p^{\prime}}} \leq C\|g\|_{L^{p^{\prime}}} \leq C
$$

Therefore

$$
\begin{aligned}
\|u\|_{L^{p}} & =\sup _{g \in H} \int u\left(\widehat{\mathcal{A}}^{*}-\lambda\right) v_{g} d x=\sup _{g \in H} \int(\widehat{\mathcal{A}}-\lambda) u v_{g} d x=\sup _{g \in H} \int f v_{g} d x \\
& \leq C|\lambda|^{-1} \mid\|f\|_{L^{p}} .
\end{aligned}
$$

Note that the duality argument implies directly the uniqueness of a variational solution of (3). We have only to prove the existence in the general case. If $f \in L^{p}$ we can find a sequence of function $f_{n} \in L^{p} \cap L^{2}$ converging to $f \in L^{p}$. By the previous estimate we have that the solutions $u_{n}$ are a Cauchy sequence in $L^{p}$. Using the regularity of the coefficients, it is not difficult to prove that the functions $u_{n}$ converge to the solution $u$ of (3) that satisfies estimate (13).

Remark 3.9. If one assumes more regular coefficients, one may characterize the domain also in the case $p<2$. Precisely for every solution $u \in D\left(\mathcal{A}_{p}\right)$ of (3) we have

or

$$
|\lambda|\|u\|_{L^{p}}+|\lambda|^{\frac{1}{2}}\|u\|_{W_{(\gamma, \psi)}^{1, p}}+\|u\|_{W_{\left(\gamma^{2}, \gamma \psi, \psi^{2}\right)}^{2, p}} \leq C\|f\|_{L^{p}}
$$

$$
|\lambda|\|u\|_{L^{1}}+|\lambda|^{\frac{1}{2}}\|u\|_{W_{(\gamma, \psi)}^{1,1}}+\|u\|_{W_{\left(\gamma^{2}, \gamma \psi\right.}^{1,1}} \leq C\|f\|_{L^{1}}
$$

according to wether $1<p<2$ or $p=1$, for every $\lambda \in \mathbb{C}$ whose real part is greater than a suitable fixed positive real number $\omega$ and for a suitable $C>0$ independent of $\lambda$.

Briefly let $f \in L^{p} \cap L^{2}$ (the general case follows as before from standard density arguments). So for every $g$ in $H$ there exists a solution $v_{g}$ of (14) and by Theorem 3.7 we have

$$
|\lambda|\left\|v_{g}\right\|_{L^{p^{\prime}}}+|\lambda|^{\frac{1}{2}}\left\|v_{g}\right\|_{W_{(\gamma, \psi)}^{1, p^{\prime}}}+\left\|v_{g}\right\|_{W_{\left(\gamma^{2}, \gamma \psi, \psi^{2}\right)}^{2, p^{\prime}}} \leq C
$$

if $1<p<2$ or

$$
|\lambda|\left\|v_{g}\right\|_{L^{\infty}}+|\lambda|^{\frac{1}{2}}\left\|v_{g}\right\|_{W_{(\gamma, \psi)}^{1, \infty}}+\left\|v_{g}\right\|_{W_{\left(\gamma^{2}, \gamma \psi\right)}^{1, \infty}} \leq C
$$

if $p=1$.

Now (15) easily follows from (17) and estimates

$$
\begin{aligned}
\|u\|_{L^{p}} & \leq\left\|v_{g}\right\|_{L^{p^{\prime}}}\|f\|_{L^{p}} \\
\|u\|_{W_{(\gamma, \psi)}^{1, p^{\prime}}} & \leq\left\|v_{g}\right\|_{W_{(\gamma, \psi)}^{1, p^{\prime}}}\|f\|_{L^{p}} \\
\|u\|_{W_{\left(\gamma^{2}, \gamma \psi, \psi^{2}\right)}^{2, p}} & \leq\left\|v_{g}\right\|_{W_{\left(\gamma^{2}, \gamma \psi, \psi^{2}\right.}^{2, p^{\prime}}}\|f\|_{L^{p}} .
\end{aligned}
$$

Similarly for (16). 
Example. Consider the PDE for the price of a European contingent claim in the multifactor case, under the so-called no-arbitrage assumption

$$
D_{t} v+\mathcal{A} v=0
$$

where

$$
\mathcal{A} v=\frac{1}{2} \operatorname{Tr}\left((\sigma \operatorname{diag} x)\left(D_{i, j} v\right)(\sigma \operatorname{diag} x)^{*}\right)+(r-\rho)(1-\epsilon) \sum_{i=1}^{d} x_{i} D_{i} v-r v
$$

with terminal condition $v(x, T)=g(x)$ (see, e.g., [18]). Here ( $\operatorname{diag} x$ ) is the diagonal matrix with the components of $x \equiv\left(x_{1}, \ldots, x_{d}\right)$ on the main diagonal, $r$ is the interest rate of a reference riskless asset in the market, $\sigma$ is a given $d$-order matrix such that, writing $\sigma^{*}$ for the transpose of $\sigma$, the matrix $\sigma^{*} \sigma$ is positive definite, $\rho \equiv \rho(x, t)$ is the dividend rate and $\epsilon \equiv \epsilon(x, t)$ is the tax rate on dividends. The solution $v \equiv v(x, t)$ represents the no-arbitrage price of a contingent claim having payoff $g \equiv g(x)$ at the expiration time $T$. In the case $d=1, \rho=0, \epsilon=0$ and $g(x)=(x-E)^{+}$, where $E$ is the maturity price of the option, we obtain the well-known Black and Scholes equation described in [4]. Also multifactor models, such as the ones appearing in [8], options on futures contracts, and swaps can be treated in our framework $([3,18])$, along with the example below.

Example. We consider here the structure model of interest rate derivatives. For the so-called affine single-term structure model the interest rate is modeled by the stochastic process $\left(X_{t}\right)_{t \geq 0}$ satisfying the differential equation

$$
d X_{t}=\left(\alpha_{1}(t)+\alpha_{2}(t) X_{t}\right) d t+\left(\beta_{1}(t)+\beta_{2}(t) X_{t}\right) d W_{t}
$$

Suitably choosing the coefficients $\alpha_{1}(t), \alpha_{2}(t), \beta_{1}(t)$ and $\beta_{2}(t)$, different termstructure models can be obtained. In particular two models fitting our framework can be obtained by choosing

1. $\alpha_{1}=\alpha_{2}=\beta_{1}=0[7]$

2. $\beta_{1}=0[5]$.

The price of a zero-coupon bond maturing at date $T$ is the solution of the Cauchy problem

$$
D_{t} v+\mathcal{A} v=0
$$

with the end terminal condition $v(x, T)=1$, where

$$
\mathcal{A} v=\frac{1}{2}\left(\beta_{1}+\beta_{2} x\right)^{2} D_{x, x} v+\left(\alpha_{1}+\alpha_{2} x\right) D_{x} v .
$$

We remark in addition that our results allow us to treat also multifactor models with time-dependent coefficients (see [8, 3]), and also semilinear perturbations of the above equations.

Example. The following equation, coming from nonlinear filtering, is considered in $[2,14]$ :

$$
D_{t}=D_{x, x}+x D_{x} v-x^{2} v, \quad t>0, x \in \mathbb{R}
$$


with initial condition $v(0, x)=g(x)$. It can be easily checked that the second order operator defined by the right-hand side of the above equation satisfies our assumptions.

\section{References}

[1] R.A. Adams, Sobolev spaces. Pure and Applied Mathematics 65. Academic Press, New York-London 1975.

[2] J.S. Baras , G.L. Blankeship and W.E.Jr. Hopkins, Existence, uniqueness and asymptotic behaviour of solutions to a class of Zakai equations with unbounded coefficients. IEEE Trans. Automat. Control 28 (1983), 203-214.

[3] E. Barucci, F. Gozzi and V. Vespri, On a semigroup approach to no-arbitrage pricing theory. Seminar on Stochastic Analysis, Random Fields and Applications, Ascona 1996, $1-14$.

[4] F. Black and M. Scholes, The pricing of options and corporate liabilities. Journal of Political Economy 81 (1973), 637-654.

[5] M. Brennan and E. Schwartz, Analyzing convertible bonds. Journal of Financial and Quantitative Analysis 17 (1982), 75-100.

[6] M. Chicco, Sulle equazioni ellittiche del secondo ordine a coefficienti continui. Ann. Mat. Pura Appl. 88 (1971), 123-133.

[7] M. Dothan, On the term structure of interest rates. Journal of Financial Economics 7 (1978), 229-264.

[8] D. Duffie, Dynamic asset pricing theory. Princeton University Press, Princeton 1996.

[9] R.A. Adams Partial differential equations. Holt, Rineheart and Winston Inc., New York-Chicago-San Francisco 1975.

[10] D. Gilbarg and N.S. Trudinger. Elliptic partial differential equations of second order. Springer-Verlag, Berlin 2001.

[11] F. Gozzi, R. Monte and V. Vespri, Generation of analytic semigroups and domain characterization for degenerate elliptic operators with unbounded coefficients arising in financial mathematics. Part I. Differential Integral Equations 15 (2002), 1085-1128.

[12] A. Lunardi, Analytic semigroups and optimality regularity in parabolic problems. Birkhäuser-Verlag, Berlin 1995.

[13] V.G. Maz'ja, Sobolev spaces. Springer-Verlag, Berlin 1985.

[14] S.J. Sheu, Solution of certain parabolic equations with unbounded coefficients and its applications. Stochastics 10 (1983), 31-46.

[15] B. Stewart, Generation of analytic semigroups by strongly elliptic operators. Trans. Amer. Math. Soc. 199 (1974), 141-162.

[16] B. Stewart, Generation of analytic semigroups by strongly elliptic operators under general boundary conditions. Trans. Amer. Math. Soc. 259 (1980), 299-310.

[17] V. Vespri, Analytic semigroups, degenerate elliptic operators and applications to nonlinear cauchy problems. Ann. Mat. Pura Appl. (4) 155 (1989), 353-388.

[18] P. Wilmott, J. Dewynne and S. Howison, Option pricing: mathematical models and computation. Oxford Financial Press, Oxford 1993. 
Massimiliano Giuli

Dipartimento di Sistemi ed Istituzioni per l'Economia

P.zza del Santuario 19

67040 Roio Poggio (AQ)

Italy

e-mail: giuli@ec.univaq.it

Fausto Gozzi

Dipartimento di Scienze Economiche e Aziendali

Viale Pola 12

00198 Roma

Italy

e-mail: fgozzi@luiss.it

Roberto Monte

Dipartimento di Studi Economico-Finanziari e Metodi Quantitativi

Via Columbia 2

00133 Roma

Italy

e-mail: monte@sefemeq. uniroma2.it

Vincenzo Vespri

Dipartimento di Matematica Ulisse Dini

Viale Morgagni 67A

50134 Firenze

Italy

e-mail: vespri@math.unifi.it 